\title{
The competition of tensor interaction and neutron-proton pairing in the beta-decay of the neutron-rich isotopes
}

\author{
E. O. Sushenok ${ }^{1, \star}$, A. P. Severyukhin ${ }^{1,2}$, N. N. Arsenyev ${ }^{1}$, and I. N. Borzov ${ }^{3,1}$ \\ ${ }^{1}$ Bogoliubov Laboratory of Theoretical Physics, Joint Institute for Nuclear Research, 141980 Dubna, Moscow \\ region, Russia \\ ${ }^{2}$ Dubna State University, 141982 Dubna, Moscow region, Russia \\ ${ }^{3}$ National Research Centre "Kurchatov Institute", 123182 Moscow, Russia
}

\begin{abstract}
The effects of the residual interaction in the particle-particle channel on $\beta$ decay characteristics and the multi-neutron emission probabilities in the $\beta$-decay of ${ }^{126,128,130,132} \mathrm{Cd}$ are studied within the quasiparticle random phase approximation with the Skyrme interaction. The coupling between one- and two-phonon terms in the wave functions of the low-energy $1^{+}$states of the daughter nuclei is taken into account. It is shown that the inclusion of the spin-isospin interaction in the particle-particle channel leads to the reduction of half-lives and redistribution of one- and two-neutron emission probabilities. The competition of tensor interaction and neutron-proton pairing in the $\beta$-decay characteristics of the neutron-rich Cd isotopes is discussed.
\end{abstract}

Study of the $\beta$-decay properties of the neutron-rich $\mathrm{Cd}$ isotopes near the neutron closed-shell $N=82$ plays the crucial role in the modeling of the astrophysical $r$-process [1-5]. The multi-neutron emission is a multistep process consisting of the $\beta$-decay of the parent nucleus $(N, Z)$ which results in feeding the excited states of the daughter nucleus $(N-1, Z+1)$ followed by the multi-neutron emissions to the ground state or $\gamma$-deexcitation to the ground state of the product nucleus $(N-1-X, Z+1)[6]$. The microscopic study of the $\beta$-decay half-lives and the $\beta$-delayed neutron-emission probabilities of the nuclei with high $N / Z$ ratio asymmetry makes it possible to reconstruct the $\beta$-decay strength function $[6,7]$.

One of the successful tools for studying the charge-exchange nuclear modes is the quasiparticle random phase approximation (QRPA) with the self-consistent mean-field derived from a Skyrme energy-density functional (EDF), see e.g., [8-11]. These QRPA calculations enable one to describe the properties of the ground state and excited charge-exchange states using the same EDF. As proposed in Ref. [12], the study of multi-neutron emission following the $\beta$-decay would be more reliable with taking into account the phonon-phonon coupling (PPC). The finite rank separable approximation (FRSA) [13-15] for the residual interaction allows one to perform such calculations in large configurational spaces.

Importance of including of density-dependent zero-range interaction in particle-particle (pp) channel was demonstrated by authors in Ref. [16], in which the properties of lowest quadrupole states in the neutron-rich nuclei near ${ }^{132} \mathrm{Sn}$ were studied within the FRSA for the Skyrme-QRPA. Role of the residual neutron-proton (np) pairing interaction in the description of the low-lying Gamow-Teller (GT)

^e-mail: sushenok@theor.jinr.ru 
strength within the QRPA-framework discussed in Ref. [8]. Recently, the $\beta$-decay characteristics of $\mathrm{Cd}$ isotopes was described [12] within the microscopic model based on the Skyrme interaction with tensor components included [17]. The inclusion of the spin-isospin interaction in the pp channel can further improves the model [18].

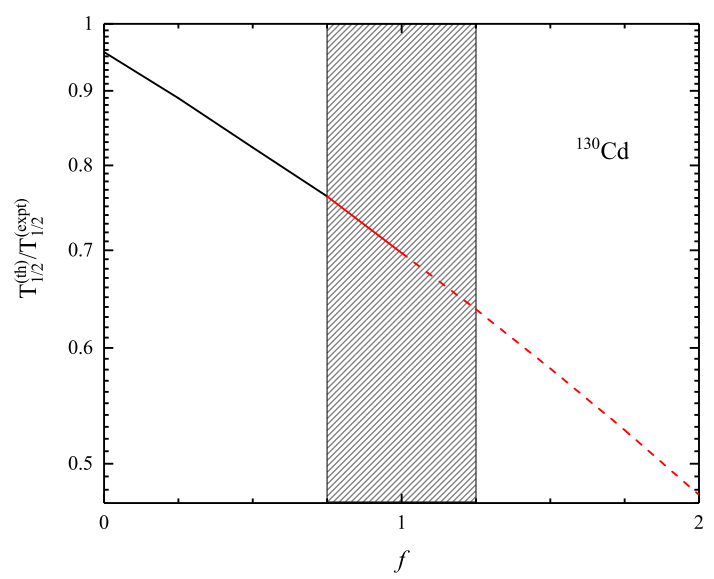

Figure 1. (Color online) The impact of dynamical neutron-proton pairing on the $\beta$-decay half-life of ${ }^{130} \mathrm{Cd}$. Calculations performed with Skyrme EDF T43 [17]. Calculated half-life is normalized to the experimental value of $T_{1 / 2}^{\text {(expt) }}=127 \pm 2 \mathrm{~ms}$ [3]. The shaded area roughly relates to the soft SU(4)-limit; dashed line corresponds to the range of the parameters, which had not been used in the presented calculations.

In this paper, we discuss the effect of the competition of tensor interaction and dynamical neutronproton pairing on the $\beta$-decay characteristics of ${ }^{126,128,130,132} \mathrm{Cd}$ isotopes. Both the central and tensor residual interactions in particle-hole ( $\mathrm{ph}$ ) channel, the neutron-proton $T=0$ pairing residual force, and the PPC effects are taken into account in the framework of FRSA model. Because the tensor force and $T=0$ pairing act in the $S=1$ channel, the effect of competition of this interactions on the low-lying GT strength is expected. In particular, Fig. 1 displays the ratio of calculated-to-experimental half-life of ${ }^{130} \mathrm{Cd}$ (see the caption for details) and demonstrates the strong dependence of the $\beta$-decay halflife on the inclusion of spin-isospin interaction in the pp channel in the case of opened-shell nuclei; see Refs. $[12,18]$. All details of the calculations, related to the study of the $\beta$-decay characteristics of the neutron-rich Cd isotopes within FRSA model, you can find in Ref. [12]. Here we focus on the part of our work devoted to the pp channel. The ground state properties of the even-even parent nucleus $(N, Z)$ described in the the Hartree-Fock-BCS (HF-BCS) model, where spherical symmetry is imposed on the quasiparticle wave functions. The continuous single-particle spectrum is discretized by diagonalizing the HF-Hamiltonian on a harmonic oscillator basis. The Hamiltonian includes the Skyrme EDF T43 [17] in the ph channel and the surface peaked density-dependent zero-range force in the pp channel

$$
\begin{aligned}
& V_{T=1}^{(\mathrm{pp})}\left(\mathbf{r}_{1}, \mathbf{r}_{2}\right)=V_{0}\left(\frac{1-P_{\sigma}}{2}\right)\left(1-\frac{\rho\left(r_{1}\right)}{\rho_{0}}\right) \delta\left(\mathbf{r}_{1}-\mathbf{r}_{2}\right), \\
& V_{T=0}^{(\mathrm{pp})}\left(\mathbf{r}_{1}, \mathbf{r}_{2}\right)=f V_{0}\left(\frac{1+P_{\sigma}}{2}\right)\left(1-\frac{\rho\left(r_{1}\right)}{\rho_{0}}\right) \delta\left(\mathbf{r}_{1}-\mathbf{r}_{2}\right)
\end{aligned}
$$


Here, $P_{\sigma}$ is the spin-exchange operator and $\rho_{0}=0.16 \mathrm{fm}^{-3}$ is the nuclear matter density. The strength $V_{0}=-870 \mathrm{MeV} \cdot \mathrm{fm}^{3}$, fixed to reproduce the odd-even mass difference of the studied nuclei [12]. The value of $f$ determines as the ratio of the strength $T=1$ and $T=0$ pairing interactions in pp channel. The np-QRPA equations are built on the basis of HF-BCS quasiparticle states of the parent (even-even) nucleus $(N, Z)[19]$. The central spin-isospin interactions in the ph channel and pp channel, derived consistently from the Skyrme EDF, can be approximated as

$$
V_{\text {res }}^{(a)}=N_{0}^{-1} G_{0}^{(a)}\left(r_{1}\right)\left(\sigma_{1} \sigma_{2}\right)\left(\tau_{1} \tau_{2}\right) \delta\left(\mathbf{r}_{1}-\mathbf{r}_{2}\right)
$$

where $a$ is the channel index $a=\{\mathrm{ph}, \mathrm{pp}\}, \tau_{i}$ is the isospin operator, and $N_{0}=2 k_{F} m^{*} / \pi^{2} \hbar^{2}$, with $k_{F}$ and $m^{*}$ standing for the Fermi momentum and nucleon effective mass. The expressions for the parameters $G_{0}^{(\mathrm{ph})}$ as well as for $G_{0}^{(\mathrm{pp})}(r)$ are

$$
\begin{aligned}
G_{0}^{\prime(\mathrm{ph})} & =-N_{0}\left[\frac{1}{4} t_{0}+\frac{1}{24} t_{3} \rho^{\alpha}+\frac{1}{8} k_{F}^{2}\left(t_{1}-t_{2}\right)\right], \\
G_{0}^{\prime(\mathrm{pp})}(r) & =\frac{1}{4} N_{0} f V_{0}\left(1-\frac{\rho(r)}{\rho_{0}}\right) .
\end{aligned}
$$

Making use of the linearized equation-of-motion approach [20] one can get the QRPA equations

$$
\left(\begin{array}{cc}
\mathcal{A} & \mathcal{B} \\
-\mathcal{B} & -\mathcal{A}
\end{array}\right)\left(\begin{array}{l}
X \\
Y
\end{array}\right)=E_{k}\left(\begin{array}{l}
X \\
Y
\end{array}\right)
$$

where the matrices $\mathcal{A}$ and $\mathcal{B}$ defined as [18]

$$
\begin{gathered}
\mathcal{A}_{a \alpha, b \beta}=-\left(u_{a} v_{\alpha} u_{b} v_{\beta}+v_{a} u_{\alpha} v_{b} u_{\beta}\right) \sum_{n, n^{\prime}=1}^{2 N+2} \kappa^{\left(n n^{\prime}\right)} d_{a \alpha}^{(n)} d_{b \beta}^{\left(n^{\prime}\right)} \\
-\left(u_{a} u_{\alpha} u_{b} u_{\beta}+v_{a} v_{\alpha} v_{b} v_{\beta}\right) \sum_{n, n^{\prime}=2 N+3}^{4 N+2} \kappa^{\left(n n^{\prime}\right)} d_{a \alpha}^{(n)} d_{b \beta}^{\left(n^{\prime}\right)}+\epsilon_{a \alpha} \delta_{a b} \delta_{\alpha \beta}, \\
\mathcal{B}_{a \alpha, b \beta}=-\left(u_{a} v_{\alpha} v_{b} u_{\beta}+v_{a} u_{\alpha} u_{b} v_{\beta}\right) \sum_{n, n^{\prime}=1}^{2 N+2} \kappa^{\left(n n^{\prime}\right)} d_{a \alpha}^{(n)} d_{b \beta}^{\left(n^{\prime}\right)} \\
+\left(u_{a} u_{\alpha} v_{b} v_{\beta}+v_{a} v_{\alpha} u_{b} u_{\beta}\right) \sum_{n, n^{\prime}=2 N+3}^{4 N+2} \kappa^{\left(n n^{\prime}\right)} d_{a \alpha}^{(n)} d_{b \beta}^{\left(n^{\prime}\right)}
\end{gathered}
$$

Here, $a, b(\alpha, \beta)$ denote the neutron (proton) quasiparticle states. The eigenvalues of the np-QRPA equations are found numerically as the roots of the FRSA secular equation [14, 15]. It enables us to perform QRPA calculations in very large two-quasiparticle spaces. It is shown that the matrix dimensions never exceed $(8 N+4) \times(8 N+4)$ independently of the configuration space size. If we omit the residual interaction in the pp channel then the matrix dimension is reduced to $(4 N+4) \times(4 N+4)$. The studies of Refs. $[14,16]$ enable us to conclude that $N=45$ is enough for the quadrupole excitations of the parent nucleus and GT transitions to the daughter nucleus.

Let us study the impact of residual interaction in the pp channel on the half-lives and neutronemission probabilities of Cd isotopes. The Table 1 demonstrates the $T_{1 / 2}$ and $P_{1 n(2 n)}$ values calculated within FRSA model, that includes the PPC effects within the Skyrme EDF T43. For the effective interaction in the pp channel, the density-dependent contact forces are used with the strength $f=1$ derived from the soft SU(4) symmetry [21, 22]. As expected, the inclusion of the spin-isospin interaction in the pp channel leads to a noticeable reduction of half-lives of Cd isotopes. It is shown that at a 
Table 1. The effect of pairing interaction on the half-lives $\left(T_{1 / 2}\right)$ and neutron-emission probabilities $\left(P_{1 n}, P_{2 n}\right)$ of $\mathrm{Cd}$ isotopes. Calculations are performed within the model, that includes the PPC effects, with (I) and without

(II) taking into account residual interaction in the pp channel. For ${ }^{130} \mathrm{Cd}$ the experimental $P_{n \text {,tot }}$ value of $3.5 \pm 1.0 \%$ [1]; in case of ${ }^{132} \mathrm{Cd}$ the experimental $P_{n, \text { tot }}$ value of $60 \pm 15 \%$ [2].

\begin{tabular}{c|ccc|ccc|c}
\hline & \multicolumn{3}{|c|}{ I } & \multicolumn{3}{|c|}{ II } & Expt. [3] \\
& $T_{1 / 2}, \mathrm{~ms}$ & $P_{1 n}, \%$ & $P_{2 n}, \%$ & $T_{1 / 2}, \mathrm{~ms}$ & $P_{1 n}, \%$ & $P_{2 n}, \%$ & $T_{1 / 2}, \mathrm{~ms}$ \\
\hline${ }^{126} \mathrm{Cd}$ & 265 & $<0.1$ & - & 166 & $<0.1$ & - & $513 \pm 6$ \\
\hline${ }^{128} \mathrm{Cd}$ & 181 & 7.1 & - & 123 & 3.7 & - & $245 \pm 5$ \\
\hline${ }^{130} \mathrm{Cd}$ & 121 & 13.5 & - & 88 & 10.5 & - & $127 \pm 2$ \\
\hline${ }^{132} \mathrm{Cd}$ & 38 & 74.8 & 25.2 & 29 & 82.0 & 18.0 & $82 \pm 4$ \\
\hline
\end{tabular}

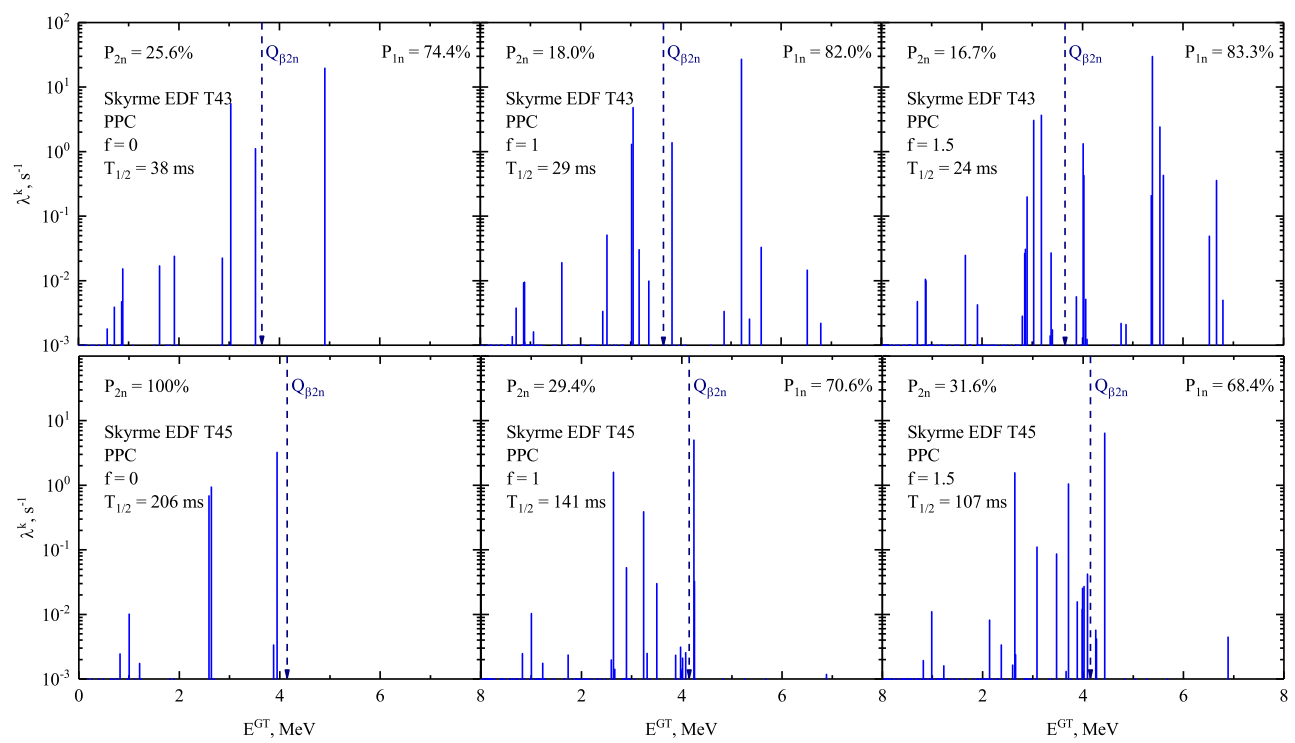

Figure 2. (Color online) The $\beta$-decay rates of ${ }^{132} \mathrm{Cd}$ calculated with Skyrme EDF's T43 (upper panels) and T45 (lower panels). The strength of parameter of $f$ is varied from 0 to 1.5 . The neutron-emission window $Q_{\beta 2 n}$ is denoted by dashed line.

qualitative level, the performed calculations describe the experimental evolution [3] of the half-lives of $\beta$-decay of neutron-rich Cd isotopes (see the Table 1).

Additional constraints on the $\beta$-strength function are given by the total and multi-neutron emission probabilities. The calculated $P_{1 n(2 n)}$ values are displayed in Table 1. As it seems, the neutron-proton pairing has the influence on the redistribution of the GT spectra of the daughter nucleus and results in the reduction of $P_{n}$ values for ${ }^{126,128,130} \mathrm{Cd}$ and $P_{2 n}$ value for ${ }^{132} \mathrm{Cd}$. It is worth mentioning that our calculations predict high probability of the two-neutron emission in the case of $\beta$-decay of ${ }^{132} \mathrm{Cd}$. This reduction can be explained by the redistribution of the strength of GT transitions (see Fig. 2, upper panel). Indeed, increasing the strength of dynamical neutron-proton pairing (2) shifts of the 
GT strength to the low-energy region. This effect is well illustrated in our calculations performed with relatively weak neutron-proton tensor interaction with respect to neutron-neutron and protonproton tensor terms (Skyrme EDF T45). The results, obtained without taking into account the residual neutron-proton pairing force $(f=0)$, predict a zero-probability of one-neutron emission for ${ }^{132} \mathrm{Cd}$.

In summary, increasing the strength of the residual $T=0$ pairing interaction leads to the significant redistribution of GT strength and results in the non-zero $P_{n}$ value and reduction of the half-life of

${ }^{132} \mathrm{Cd}$. It is shown that the competition tensor interaction and neutron-proton pairing has a substantial effect on the distribution of the Gamow-Teller transition strength. Decreasing the strength of neutronproton tensor interaction amplifies the impact of neutron-proton pairing on the beta-decay rates.

\section{Acknowledgments}

This work is supported by the Russian Science Foundation (Grant No. RSF-16-12-10161).

\section{References}

[1] M. Hannawald et al. (ISOLDE Collaboration), Nucl. Phys. A 688, 578c (2001)

[2] I. Dillmann et al. (ISOLDE Collaboration), Phys. Rev. Lett. 91, 162503 (2003)

[3] G. Lorusso et al., Phys. Rev. Lett. 114, 192501 (2015)

[4] R. Dunlop et al., Phys. Rev. C 93, 062801(R) (2016)

[5] A. Jungclaus et al., Phys. Rev. C 94, 024303 (2016)

[6] I. N. Borzov, Phys. Rev. C 71, 065801 (2005)

[7] I. N. Borzov, EPJ Web of Conf. 107, 06002 (2016)

[8] J. Engel, M. Bender, J. Dobaczewski, W. Nazarewicz, R. Surman, Phys. Rev. C 60, 014302 (1999)

[9] M. Bender, J. Dobaczewski, J. Engel, W. Nazarewicz, Phys. Rev. C 65, 054322 (2002)

[10] S. Fracasso, G. Colò, Phys. Rev. C 76, 044307 (2007)

[11] C. L. Bai, H. Sagawa, H. Q. Zhang, X. Z. Zhang, G. Colò, F. R. Xu, Phys. Lett. B 675, 28 (2009)

[12] A. P. Severyukhin, N. N. Arsenyev, I. N. Borzov, E. O. Sushenok, Phys. Rev. C 95, 034314 (2017).

[13] Nguyen Van Giai, Ch. Stoyanov, V. V. Voronov, Phys. Rev. C 57, 1204 (1998)

[14] A. P. Severyukhin, V. V. Voronov, Nguyen Van Giai, Prog. Theor. Phys. 128, 489 (2012)

[15] A. P. Severyukhin, H. Sagawa, Prog. Theor. Exp. Phys. 2013, 103D03 (2013)

[16] A. P. Severyukhin, V. V. Voronov, Nguyen Van Giai, Phys. Rev. C 77, 024322 (2008)

[17] T. Lesinski, M. Bender, K. Bennaceur, T. Duguet, J.Meyer, Phys. Rev. C 76, 014312 (2007)

[18] E. O. Sushenok, A. P. Severyukhin, N. N. Arsenyev, I. N. Borzov, Phys. At. Nucl., in press

[19] J. Terasaki, J. Engel, M. Bender, J. Dobaczewski, W. Nazarewicz, M. Stoitsov, Phys. Rev. C 71, $034310(2005)$

[20] D. J. Rowe, Nuclear Collective Motion: Models and Theory (Methuen, London, 1970)

[21] E. Wigner, Phys. Rev. 51, 106 (1937)

[22] Yu. V. Gaponov, Yu. S. Lyutostanskii, Sov. J. Part. Nucl. 12, 528 (1981) 